\title{
A Nonuniform Spatial Rain Attenuation Model for Troposcatter Communication Links
}

\author{
Ergin Dinc, Student Member, IEEE, Ozgur B. Akan, Senior Member, IEEE
}

\begin{abstract}
Troposcatter communication can be used as a communication medium for Beyond-Line-of-Sight (BLOS) links. However, wave propagation in troposphere shows significant dependence on hydro-meteors: especially rain. Therefore, the main motivation of this paper is to develop a rain attenuation model for troposcatter communications which can model nonuniform multiple rain cells for the first time. At the end, we present simulation results for the amount of rain loss and the distribution of maximum data rates under rain in troposcatter links.
\end{abstract}

Index Terms-Troposcatter, Channel characterization and modeling, Rain attenuation, Beyond-Line-of-Sight (BLOS)

\section{INTRODUCTION}

$\mathbf{T}$ ROPOSCATTER communications utilize scattering effect of lower troposphere to provide direct communication at BLOS ranges up to $300 \mathrm{~km} \mathrm{[1]-[3].} \mathrm{Troposcatter}$ communication has high losses due to high path lengths and scattering. Therefore, path lengths should be minimized by making elevation angles of antennas as small as possible. In this way, troposcatter common volume, which is the intersection of transmit and receive antenna beams formed at the lower troposphere, and scattered rays are exposed to the effects of hydro-meteors. Since modern troposcatter equipments utilize frequency range between $4.7-15 \mathrm{GHz}$ to provide more bandwidth, the most important hydro-meteor for troposcatter communications becomes rain because the effects of cloud and fog become considerable for carrier frequencies higher than 50 $\mathrm{GHz}$ [4].

There are many rain attenuation models in the literature [5]-[8]. Many of them consider rain attenuation in satellite communications (SATCOM) [7], [8]. Since the geometry of SATCOM is different compared to troposcatter, rain attenuation models for SATCOM cannot be used for troposcatter communications. In addition, the most relevant study for our work is provided by International Telecommunication Union (ITU) [5] in which rain attenuation in troposcatter links is modeled with a rectangular rain cell having a uniform rainfall distribution and located in the middle of communication path. However, distribution of rainfall rate in horizontal plane shows significant changes in the real world conditions [9]. In addition, troposcatter paths cover $50-300 \mathrm{~km}$ ranges, thus it is very high likely that the link is exposed to multiple rain cells.

The main contribution of this paper is to develop a rain attenuation model for troposcatter communications which con-

E. Dinc and O. B. Akan are with the Next-generation and Wireless Communications Laboratory, Department of Electrical and Electronics Engineering, Koc University, Istanbul, 34450 Turkey (e-mail: edinc@ku.edu.tr; akan@ku.edu.tr). siders distribution of rainfall rates and multiple rain cells for the first time. Therefore, we develop rain path length expressions for nonuniform rainfall distribution and calculate rain loss due to each rain cell. We utilize available rain models [9], [10] to generate synthetic rain distribution. We simulate the proposed rain attenuation model by modifying our previously introduced ray-based troposcatter channel modeling approach [2] to predict rain loss and maximum data rates in the presence of rain for the first time.

\section{RAY-BASED APPROACH}

In the ray-based approach, path length and power of each ray is calculated separately to form power delay spectrum (PDS) [2]. In this way, each ray is subjected to different conditions according to its own path. In this approach, differential bistatic radar range equation is given as

$$
P_{r}=\frac{P_{t} G_{t} G_{r} \sigma_{V} \lambda^{2} \varrho a_{\text {rain }}}{(4 \pi)^{3} R_{t}^{2} R_{r}^{2}} \mathrm{~W},
$$

where $P_{t}$ is the transmitter power, $\lambda$ is the wavelength, $G_{t, r}$ are the TX/RX antenna gains, $R_{t, r}$ is the distance between the scatterer to TX/RX [11]. $\varrho$ is the polarization mismatch factor. $\sigma_{V}$ is the differential scattering cross-section which is modeled with the Rayleigh scattering approximation [2], [12]. $a_{\text {rain }}$ is the rain attenuation which is introduced in this paper. With the ray-based approach, rain attenuation associated with each ray is calculated separately according to distribution of rainfall rates. In this paper, rain attenuation is mostly presented in $\mathrm{dB}$ as $A_{\text {rain }}$.

\section{Rain Cell Model}

We utilize HYCELL model [9], [10] to generate rain cells and their spatial distribution. However, we slightly modify HYCELL to integrate with our ray-based method and rain attenuation model. In [9], HYCELL utilizes both Gaussian and exponential function to characterize rainfall distribution within a rain cell. Since rainfall rates that are associated with exponential function is too low, we model the rain cells only with Gaussian component. Therefore, rainfall rate distribution in a single cell is found as

$$
R(x, y)= \begin{cases}R_{G} \exp \left[-\left(x^{2} / a_{G}^{2}+x^{2} / b_{G}^{2}\right)\right], & \text { if } R>R_{1} \\ 0, & \text { otherwise }\end{cases}
$$

where $R_{G}$ represents the peak rainfall rate. $a_{G}, b_{G}$ are the distance where the rainfall rate decreases by a factor of $1 / e$ in $\mathrm{x}$ and y axis. We assume symmetrical rain cells $a_{G}=b_{G}$. $R_{1}$ is the minimum rain rate, and it is assumed as $1 \mathrm{~mm} / \mathrm{hr}$ as 


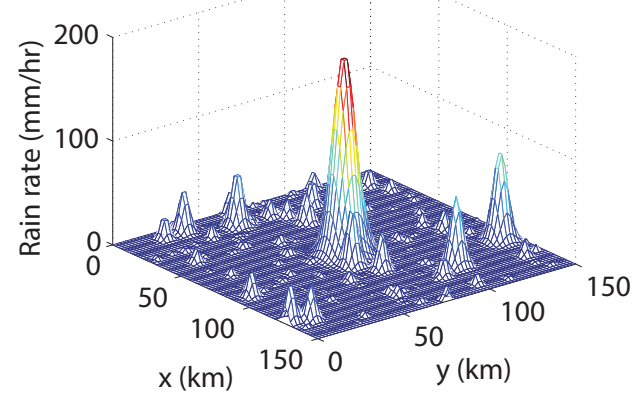

(a)

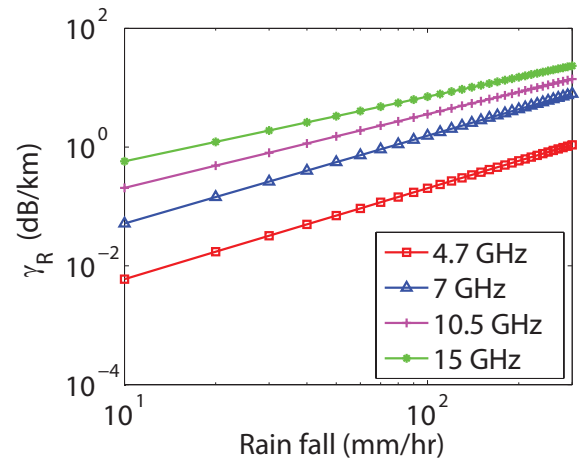

(b)

Fig. 1. (a) Spatial distribution of rain cells and, (b) rain specific attenuation.

in [9]. Spatial distributions of rain cells are determined based on local spatial probability of rain, and we utilize ITU-R P. $837-6$ [13] for local rain statistics. With HYCELL method [9], [10], we derive peak rain rates and diameters of rain cells for Istanbul, Turkey [13] as in Figure 1(a) for $150 \times 150 \mathrm{~km}^{2}$ region.

In HYCELL model, spatial distribution of rain cells is studied, but rain height is not discussed. In this paper, maximum rain height is critical to calculate rain attenuation in troposcatter links. As in Figure 2, rectangular rain cells are modeled in propagation path and only portion of rays which passes through a rain cell is affected by rain. Therefore, height of a rain cell $\left(h_{R}\right)$ is determined by [6] $h_{R}=h_{0}+0.36 \mathrm{~km}$, where $h_{0}$ is the mean $0^{\circ} \mathrm{C}$ isotherm height above mean sea level and average values for this parameter is used as provided in [6].

\section{Rain AtTenuation Model}

Scattering and absorbing effects of rain droplets degrade performance of the troposcatter communications. Therefore, we propose a rain attenuation model for troposcatter communication. Unlike scattering particles $(1-10 \mu \mathrm{m})$, sizes of rain droplets can vary $1 \mu \mathrm{m}$ up to $1 \mathrm{~mm}$. Since size of rain droplets and wavelength of signals become comparable especially for high carrier frequencies between $10-15 \mathrm{GHz}$, the effect of rain droplets cannot be modeled by Rayleigh scattering approximation as in [2]. To this end, in the presence of rain, path loss calculations require a correction factor $(S)$ for the Rayleigh scattering approximation when carrier frequency is higher than $10 \mathrm{GHz}$ and, this correction factor in $\mathrm{dB}$ is given as [5]

$$
S= \begin{cases}R_{G}^{0.4} 10^{-3}\left[4(f-10)^{1.6}((1+\cos (\Psi)) / 2)+\right. \\ \left.+5(f-10)^{1.7}((1+\cos (\Psi)) / 2)\right], & f>10 \mathrm{Ghz} \\ 0, & f \leq 10 \mathrm{Ghz}\end{cases}
$$

where $R_{G}$ is the peak rainfall rate $(\mathrm{mm} / \mathrm{hr}), f$ is the frequency $(\mathrm{GHz})$, and $\Psi$ is the scatter angle.

\section{A. Rain Specific Attenuation}

Rain specific attenuation $\left(\gamma_{R}\right)$, that is amount of path loss in $\mathrm{dB}$ per $\mathrm{km}$ due to rain, is [14]

$$
\gamma_{R}=k R^{\gamma} \mathrm{dB} / \mathrm{km},
$$

where $k$ and $\gamma$ are empirical coefficients that show dependence on carrier frequency, path elevation angle and polarization. Empirical values for these coefficients can be found in [14]. Figure 1(b) shows rain specific attenuation as a function of rainfall rate for different carrier frequencies by using (4) and the parameters given by [14]. As noticed, high frequencies show more dependence on rain attenuation because effects of rain droplets increases as sizes of rain droplets and wavelength become more comparable.

\section{B. Rain Path Calculations}

Rain attenuation depends on rain cell geometry as in Figure 2 and 3. Therefore, rain path calculations should be performed separately for each path to include the effects of rain cell geometry. For troposcatter channel, rain path calculations are proposed in [5], but this model assumes a single rain cell at the middle of a troposcatter link with uniform rainfall rate distribution. As a result, this model is unrealistic for the real world conditions. Therefore, we develop rain path calculations for rain cells which can be anywhere between transmitter and receiver.

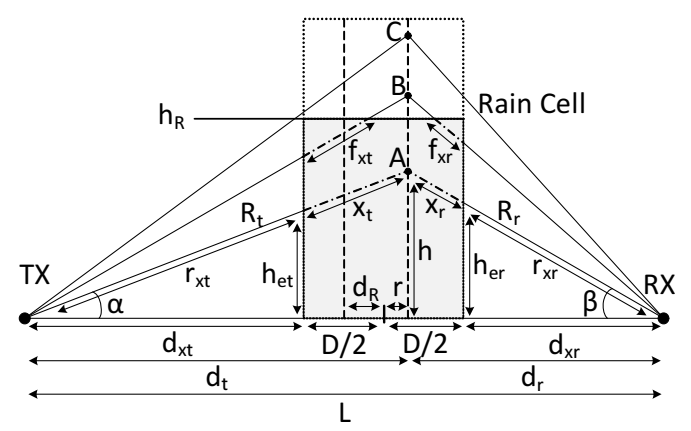

Fig. 2. Geometry of the rain cell for Category 1.

As in Figure 2 and 3, troposcatter rays can be affected from rain conditions in different ways according to place of rain cell and scattering point. As a result, we divide rays into two main category according to location of rain cells. In the first category, distance between center of the rain cell and center of the communication path $\left(d_{R}\right)$ is smaller than rain width 
$D / 2$ as in Figure 2. In the second category $\left(d_{R}>D / 2\right)$, only one side of path is affected by rain cell as shown in Figure 3 . To include multiple rain cells, rain attenuation calculations should be performed for each rain cell separately. At the end, total rain attenuation is given as

$$
A_{\text {rain }}^{\text {Total }}=\sum_{i=1}^{N_{R}} A_{\text {rain }}^{i} \mathrm{~dB},
$$

where $N_{R}$ is the number of rain cell, $A_{\text {rain }}^{i}$ is the rain attenuation associated with rain cell $i$.

1) Category $1\left(d_{R}<D / 2\right)$ : As in Figure 2, path calculations for this category follow as:

Lower Paths A: Lower paths (path $\mathbf{A}$ in Figure 2) have scattering point within the rain cell. Rain cell path lengths for transmitter side $\left(x_{t}\right)$ and receiver side $\left(x_{r}\right)$ are found as

$$
\begin{aligned}
d_{t, r} & =R_{t, r} \cos (\alpha, \beta), r=d_{t}-L / 2-d_{R}, \\
d_{x_{t, r}} & =d_{t, r}-D / 2 \mp r, r_{x_{t, r}}=d_{x_{t, r}} / \cos (\alpha, \beta), \\
x_{t, r} & = \begin{cases}R_{t, r}-r_{x_{t, r}} & d_{t, r}>D / 2 \\
R_{t, r} & d_{t, r}<D / 2 .\end{cases}
\end{aligned}
$$

Rain attenuation for lower paths at transmitter $\left(A_{\operatorname{rain} T}\right)$ is found by taking the integral of (4) for nonuniform rainfall distribution in (2) over the rain path calculated in (6) as

$$
\begin{aligned}
A_{\text {rain } T} & =\int_{-\frac{D}{2}}^{\frac{x_{t}}{\cos (\alpha)}-\frac{D}{2}} k R(x)^{\gamma} \frac{1}{\cos (\alpha)} d x \mathrm{~dB}, \\
& =\int_{-\frac{D}{2}}^{\frac{x_{t}}{\cos (\alpha)}-\frac{D}{2}} \frac{R_{G}^{\gamma}}{\cos (\alpha)} \exp \left(-\frac{x^{2}}{a_{G}^{2}}\right)^{\gamma} d x \mathrm{~dB} .
\end{aligned}
$$

With the same method, rain attenuation for receiver side is found as

$$
A_{\text {rain } R}=\int_{\frac{D}{2}-\frac{x_{r}}{\cos (\beta)}}^{\frac{D}{2}} R_{G}^{\gamma} / \cos (\beta) \exp \left(-x^{2} / a_{G}^{2}\right)^{\gamma} d x \mathrm{~dB}
$$

Total rain attenuation in each ray is the summation of (3), (7) and (8) as $A_{\text {rain }}=S+A_{\text {rain } T}+A_{\text {rain } R} \mathrm{~dB}$.

Middle Paths B: Scattering point of middle paths (associated with $\mathbf{B}$ in Figure 2) are outside of the rain cell and also some portion of the rays is still inside. Therefore, path lengths for middle paths is given as

$$
f_{x_{t, r}}= \begin{cases}\left(h_{R}-h_{e_{t, r}}\right) / \sin (\alpha, \beta), & h_{e_{t, r}}<h_{R} \\ 0 . & \text { otherwise. }\end{cases}
$$

where $h_{e_{t, r}}=d_{x_{t, r}} \tan (\alpha, \beta)$

In addition to rain attenuation, scattering cross-section decreases as a function of height above rain height with -6.5 $\mathrm{dB} / \mathrm{km}$ [5]. Therefore, total rain attenuation for middle paths is given as

$$
\begin{aligned}
A_{\text {rain }} & =6.5\left(h-h_{r}\right)+\int_{-\frac{D}{2}}^{\frac{f_{x_{t}}}{\cos \alpha}-\frac{D}{2}} \frac{R_{G}^{\gamma}}{\cos (\alpha)} \exp \left(-\frac{x^{2}}{a_{G}^{2}}\right)^{\gamma} d x \\
& +\int_{\frac{D}{2}-\frac{f_{x_{r}}}{\cos \beta}}^{\frac{D}{2}} \frac{R_{G}^{\gamma}}{\cos (\beta)} \exp \left(-\frac{x^{2}}{a_{G}^{2}}\right)^{\gamma} d x+S \mathrm{~dB} . \quad \text { (10) }
\end{aligned}
$$

Upper Paths C: Upper paths, that are associated with $\mathbf{C}$ in Figure 2, have no parts in rain cell. However, since scattering cross-section decreases above rain height as in middle paths, rain loss in upper paths is determined as

$$
A_{\text {rain }}=S+\exp \left(-k_{r}\left(6.5\left(h-h_{r}\right)\right) \mathrm{dB} .\right.
$$

2) Category $2\left(d_{R}>D / 2\right)$ : Category 2 rays are associated with rain cells on only one side as in Figure 3. Path calculations for this category are given as:

Lower Paths D: In category 2, lower paths are associated with $\mathbf{D}\left(h_{e_{t, r}}<h_{R}\right)$ in Figure 3. Slant paths inside rain cells are found as

$$
\begin{aligned}
& x_{t}= \begin{cases}D / \cos (\alpha), & h_{e_{r}}>h_{e_{t}} \\
0, & \text { otherwise }\end{cases} \\
& x_{r}= \begin{cases}D / \cos (\beta), & h_{e_{r}}<h_{e_{t}} \\
0, & \text { otherwise }\end{cases}
\end{aligned}
$$

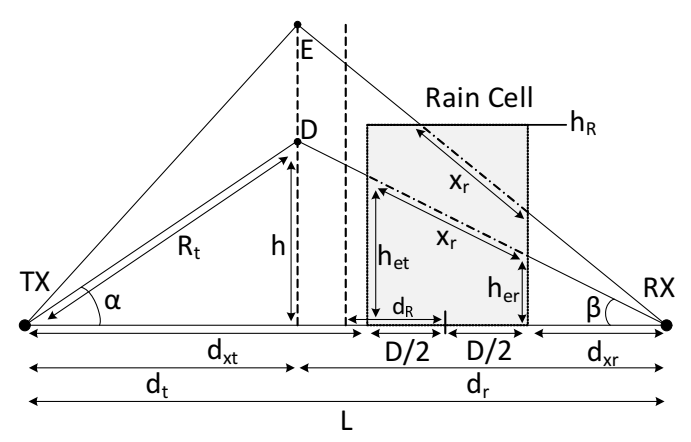

Fig. 3. Geometry of the rain cell for Category 2 .

Middle Paths E: In category 2, middle paths are associated with $\mathbf{E}\left(h_{e_{t}}>h_{R}\right.$ or $\left.h_{e_{r}}>h_{R}\right)$ in Figure 3. Rain cell path lengths for path $\mathbf{E}$ can be found as

$$
\begin{aligned}
& x_{t}= \begin{cases}\left(h_{R}-h_{e_{t}}\right) / \sin (\alpha), & h_{e_{r}}>h_{e_{t}} \\
0, & \text { otherwise }\end{cases} \\
& x_{r}= \begin{cases}\left(h_{R}-h_{e_{r}}\right) / \sin (\beta), & h_{e_{r}}<h_{e_{t}} \\
0, & \text { otherwise }\end{cases}
\end{aligned}
$$

For paths $\mathbf{D}$ and $\mathbf{E}$, resulting rain attenuation expressions are given as

$$
\begin{aligned}
A_{\text {rain }} & =6.5\left(h-h_{r}\right)+\int_{-\frac{D}{2}}^{\frac{x_{t}}{\cos \alpha}-\frac{D}{2}} \frac{R_{G}^{\gamma}}{\cos (\alpha)} \exp \left(-\frac{x^{2}}{a_{G}^{2}}\right)^{\gamma} d x \\
& +\int_{\frac{D}{2}-\frac{x_{r}}{\cos \beta}}^{\frac{D}{2}} \frac{R_{G}^{\gamma}}{\cos (\beta)} \exp \left(-\frac{x^{2}}{a_{G}^{2}}\right)^{\gamma} d x+S \mathrm{~dB} . \quad \text { (14) }
\end{aligned}
$$

Upper paths for category 2 is the same as category 1 . Thus, (11) is also valid for category 2 .

\section{Maximum Data Rate}

Maximum data rate in single-input-single-output troposcatter links can be calculated as

$$
C(t)=B \log _{2}\left(1+P_{r}(t) / N\right) \text { bps, }
$$

where $P_{r}$ is the received power. $N$ is the noise power (modeled as thermal noise $N=k B T$ ), $T$ is the temperature in $\mathrm{K}, B$ is the bandwidth and $k$ is the Boltzmann constant. 


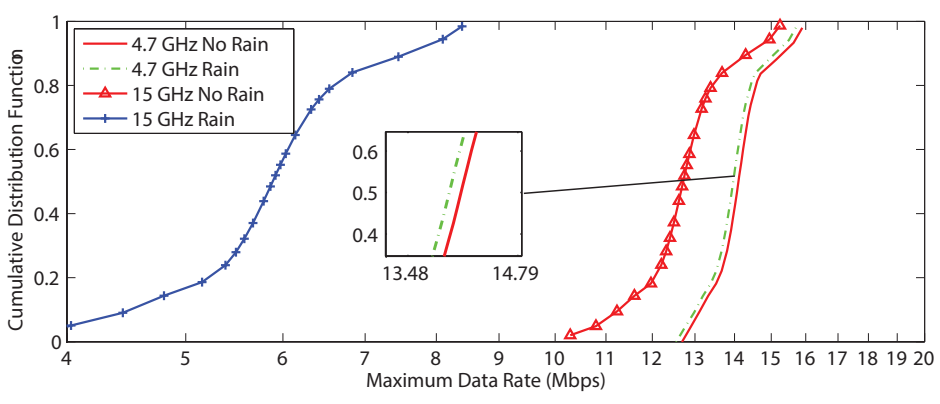

(a)

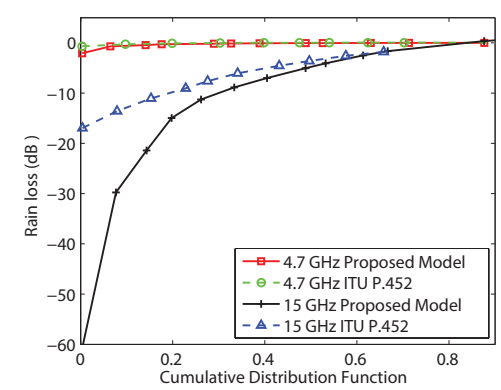

(b)

Fig. 4. (a) Distribution of data rates under rain for different carrier frequencies, (b) CDF of rain loss.

Our ray-based method [2] utilizes the real-world water vapor mixing ratio measurements [15]. Therefore, this approach can model fluctuations in the channel. In this work, we also utilize the same data sets [15] to model the atmospheric variations in the presence of rain to derive data rates and rain loss results. This approach [2] is also utilized in [3] to derive data rates for diversity techniques. In this way, PDS of the channel is calculated for each measurement instant with (1) and the path length calculations in [2], [11]. Average power of PDS for each time instant is denoted as $P_{r}(t)$ in (15). The effect of rain attenuation is integrated to the ray-based approach with $a_{\text {rain }}$ in (15) to calculate data rates and rain loss in the presence of rain.

\section{Simulation Results}

In the ray-based approach, rain attenuation for each ray is calculated with the troposcatter path length calculations for the rainfall distribution as in Figure 1(a). The rain simulations are performed for each cross-section of Figure 1(a) for each $500 \mathrm{~m}$ to find the CDF of rain loss for the modeled region on MATLAB. The following channel parameters are utilized: range $L=150 \mathrm{~km}$, beam elevation of antennas $\alpha_{0}=\beta_{0}=8.3$ mrad, bandwidth $B=1 \mathrm{MHz}$. For frequency dependent terms, we utilize commercial troposcatter equipment data-sheets [16]: $G_{t}=G_{r}=38.3 \mathrm{~dB}$, antenna beam-widths $2.08^{\circ}$, transmit power $P_{t}=1000 \mathrm{~W}$ for $4.7 \mathrm{GHz}$ and $G_{t}=G_{r}=49.7 \mathrm{~dB}$, antenna beam-widths $0.54^{\circ}$, transmit power $P_{t}=250 \mathrm{~W}$ for $15 \mathrm{GHz}$.

Figure 4(a) shows the distributions of the maximum data rates for different frequencies with and without rain by using the real world data sets as discussed in Section V. The amount of rain loss increases with carrier frequency because the wavelengths and the sizes of the rain droplets becomes more comparable with the increasing carrier frequency. As in Figure 4(a), only extreme rain conditions can affect the performance of the $4.7 \mathrm{GHz}$ curves. However, $15 \mathrm{GHz}$ show very high dependence on rain. The same result can be seen in Figure 4(b) which presents distribution of rain loss with the proposed rain model and the single cell model in ITU P.452 [5]. As expected, the rain loss estimates with the single cell model are considerably lower especially at high frequencies because the single cell model cannot model the effects of multiple rain cells. In addition, $4.7 \mathrm{GHz}$ shows slight dependence on rain attenuation, but there may be $3 \mathrm{~dB}$ power loss in the link. Therefore, we can conclude that lower carrier frequencies are more promising for troposcatter communication links thanks to their resistance to the rain conditions.

\section{CONCLUSION}

In this paper, we developed a rain attenuation model to predict the amount of rain loss in troposcatter links for non-homogeneous rain cells. According to our results, low carrier frequencies do not show significant changes with rain. However, high carrier frequencies show significant rain losses. Therefore, lower frequencies are more promising for troposcatter applications.

\section{REFERENCES}

[1] L. Bastos, H. Wietgrefe, "Tactical troposcatter applications in challenging climate zones," MILCOM 2012, pp. 1-6, 2012.

[2] E. Dinc, O. B. Akan, "A Ray-based Channel Model For MIMO Troposcatter Communications," Proc. IEEE PIMRC 2013, September 2013.

[3] E. Dinc, O. B .Akan, "Fading Correlation Analysis in MIMO-OFDM Troposcatter Communications: Space, Frequency, Angle and Spacefrequency Diversity," to be published in IEEE Transactions on Communications, 2015.

[4] "ITU-R's Rec. P. 840-4: Attenuation Due To Clouds And Fog," 2009.

[5] "ITU-R's Rec. P. 452-15: Prediction procedure for the evaluation of interference between stations on the surface of the Earth at frequencies above about $0.1 \mathrm{GHz}, " 2013$.

[6] "ITU-R's Rec. P. 839-4: Rain height model for prediction methods," 2013.

[7] Crane, R. K., Electromagnetic Wave Propagation Through Rain, Wiley Ser. Remote Sens., John Wiley, New York, 1996.

[8] A. Dissanayake, J, Allnutt, F. Haidara, "A prediction model that combines rain attenuation and other propagation impairments along Earth-satellite paths," IEEE Trans. Antennas Propag., vol. 45, no. 10, Oct. 1997.

[9] L. Feral, H. Sauvageot, L. Castanet, J. Lemorton, "HYCELL - A new hybrid model of the rain horizontal distribution for propagation studies: 1. Modeling of the rain cell," Radio Science, vol. 38, no. 3, 2003.

[10] Feral L., Sauvageot H., Castanet L., Lemorton J., "HYCELL - A new hybrid model of the rain horizontal distribution for propagation studies: 2. Statistical modeling of the rain rate field," Radio Science, vol. 38, no. 3, 2003.

[11] G. Roda, Troposcatter Radio Links. Artech House Publishers, 1988.

[12] A. Ishimaru, Wave Propagation and Scattering in Random Media. IEEE Press Series on Electromagnetic Wave Theory, Wiley, 1999.

[13] "ITU-R's Rec. P. 837-6: Characteristics of precipitation for propagation modeling," ITU, Feb. 2012.

[14] “ITU-R's Rec. P. 838-3: Specific attenuation model for rain for use in prediction methods," ITU, 2005.

[15] NAMMA LASE, NASA EOSDIS GHRC DAAC, [online] Available: http://ghrc.msfc.nasa.gov/index.html. Accessed on Dec 22, 2014.

[16] Troposcatter, General Dynamics SATCOM Technologies," [online], http://www.gdsatcom.com/troposcatter.php. (Accessed: 23 Apr. 2014). 